\title{
A Case of Sub-acute Combined Degeneration of the Spinal Cord with Associated Pernicious Anaemia
}

\author{
A A Bhuiyan ${ }^{1}$, S K Dash², S M H Shahriar ${ }^{3}$, F Nahid ${ }^{4}$, S Arefin ${ }^{5}$
}

\begin{abstract}
Aim and Objective

Vitamin B12 deficiency disease, specially associated with pernicious anaemia is a relatively rare disease in the developing countries. Patients with B12 deficiency may present with hematological, gastro-intestinal and neuro-psychiatric manifestations. Here we discuss a case of a fifty five-year-old lady presented with sub-acute combined degeneration of the spinal cord.

\section{Case presentation}

A fifty five year old female was admitted in Neurology ward in Apollo Hospitals, Dhaka from OPD for progressive quadriparesis with tingling in the hands and feet. She had no associated visual, bulbar symptoms, sphincter incontinence or memory impairment. Investigation revealed mild anaemia, macrocytosis on peripheral blood picture, low Vitamin B12 level with megaloblastic changes in bone marrow examination. Anti-Intrinsic factor antibody and anti-parietal cell antibody was not done, as it is not available here. MRI of dorsal spine shows T2 hyper-intense lesions in the posterior cord. GI Endoscopic biopsy revealed chronic atrophic gastritis.
\end{abstract}

\section{Conclusion}

We presented this case because of its relatively uncommon occurrence in our country. Sub-acute combined degeneration of spinal cord associated with dietary deficiency is common in Indian sub-continent. High index of suspicion is needed for its early diagnosis as delay in treatment can lead to poor neurological recovery.

\section{Key word}

Vitamin B12, sub-acute combined degeneration, pernicious anaemia.

\section{Introduction}

The neuro-psychiatric manifestation of Vitamin B12 deficiency involves the brain, spinal cord, optic nerve and peripheral nervous system. Sub-acute combined degeneration of the spinal cord is the most serious neurological complication of vitamin B12 deficiency and most commonly occurs with megaloblastic anemia.

Symptoms of anemia usually precede the neurological manifestations. Nonetheless, the median time to diagnose from symptom onset in neurological presentation of B12 deficiency in general is approximately one year; with a range extending to more than four years. ${ }^{1}$ The neurological complication affected twenty to forty percent of earlier reported cases of pernicious anemia. ${ }^{2}$

\section{Case report}

A fifty five year old female was admitted in AHD in November, 2010 with progressive weakness and stiffness in the lower extremities over the last 1 year. For last two months she had difficulty in walking and needed a cane to walk. 
Three to four months prior to the onset of this symptom, she started having tingling and paraesthesia in her fingertips which later spread to involve her feet distally. She had no visual, bulbar symptoms, sphincter incontinence or noticeable cognitive impairment. There was no associated fever, weight loss and pain in the spine or limbs. She was a non-smoker, non-alcoholic and had a reasonably balanced diet. She had no history of mal-absorption or any gastro-intestinal surgery. She had no exposure to toxins, chemicals and was not taking lentils (Kasseri). She was not taking any medications including those which can decrease S.Vit. B12 level. There was no family history of any neuro-muscular disease.

Her vital signs were normal, systemic examination revealed mild anemia, glossitis (Figure-1), vitiligo over lips, lower abdomen (Figure-2) and lower limbs.

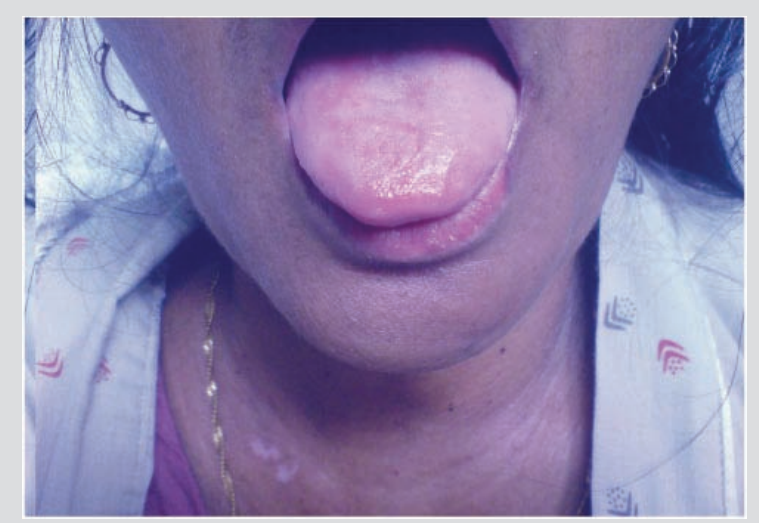

Figure 1: Glossitis

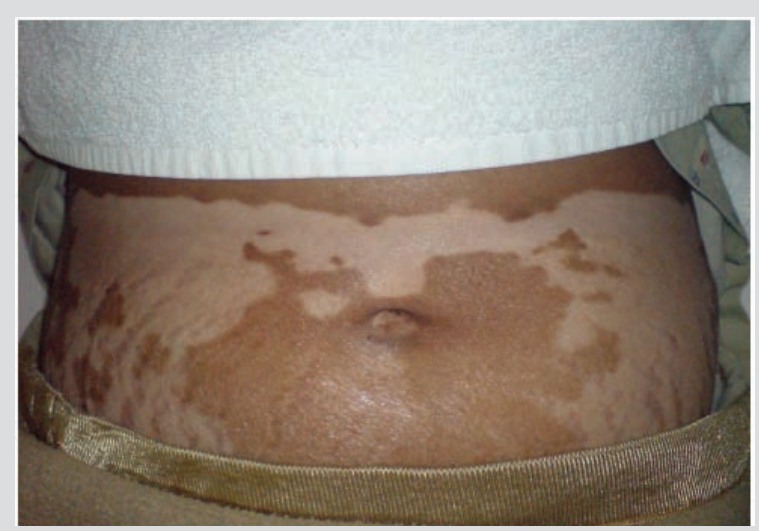

Figure 2: Vitiligo over lower abdomen
Neurological examination showed mild cognitive impairment (27/30 on MMS).

Cranial nerve exam showed visual acuity was $6 / 60$ in right eye, $3 / 60$ in left eye and fundoscopy showed bilateral temporal disc pallor without evidence of any papillodema. There was no afferent papillary defect, nystagmus or facial asymmetry.

Motor exam showed power of $4 / 5$ in upper limbs, 3/5 in lower limbs with spasticity noted especially in lower extremities; without evidence of any muscle atrophy.

Her sensory examination is significant for gross impairment of position and vibration sense, especially in lower extremities with preserved fine touch and pin-prick sensation. Romberg test was positive. There was no sensory level.

Reflex exam showed normal reflexes in upper extremities and hyper-reflexia in the knees, ankles with an equivocal planter responses. There was no ankle clonus.

Her gait was slow and spastic. She needed one persons help to walk. She had normal cardiac, respiratory and gastro-intestinal examination.

Laboratory investigation reveals hemoglobin of $12.4 \mathrm{gm} / \mathrm{dl}$ with raised MCV (108.0 fl).

Peripheral blood smear showed macrocytic blood picture.

Serum Vitamin B-12 was low $<150$ pg/ml (220-915).

Random Blood Sugar, TSH, folate, LFT, LDH, creatinine were all normal.

Bone marrow examination was hyperactive with megaloid changes.

Subsequent GI Endoscopic biopsies showed mild to moderate glandular atrophy along with extensive infiltration of lamina propria by lymphoplasmacytic cells and eosinophils which was consistent with chronic atrophic gastritis.

MRI Scan of the brain was normal. MRI Scan of the Dorsal Spine showed $T_{2}$ hyper intense lesions in the dorsal column of upper dorsal spine (Figure-3). 


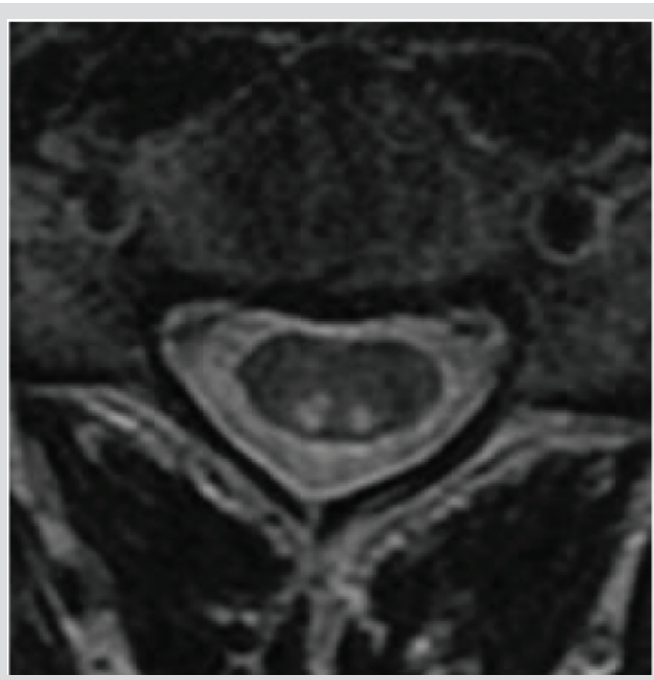

Figure 3: $T_{2}$ hyper intense lesions in the dorsal column of upper dorsal spine

Patient was given Injection Vitamin B12 1mg I/M daily for 7 days and weekly for 1 month and other supportive treatment. She was advised to continue on monthly injections for the rest of her life. She was prescribed eye-glasses by Consultant Ophthalmologist for her refractive error. Follow up after one month, she showed significant improvement in her motor strength. Now she could get up from squatting position and walk without help which she could not do earlier. Sensory impairment showed some improvement in proprioception. There was no appreciable change in her cognitive function. Her lesion in the tongue was nearly resolved. Vitamin B12 level increased to over 1000 picogram/dl.

\section{Discussion}

This patient as described presented with features consistent with sub-acute combined degeneration of the spinal cord. The presentation was slowly progressive spastic paraparesis, proprio ceptive loss with mild cognitive impairment without any optic nerve involvement. MRI of dorsal spine showed T-2 hyper-intense lesions only in the dorsal column without any compressive or inflammatory lesions. There was no family history of hereditary spastic paraperesis.

The patient's typical presentation, glossitis, vitiligo and biopsy evidence of chronic atrophic gastritis is highly suggestive of auto-immune disease (pernicious anaemia), although anti-intrinsic factor antibody and anti-parietal cell antibody was not done due to its non-availability.

Sub-acute combined degeneration of the spinal cord due to dietary deficiency is rare in Bangladesh due to consumption of sufficient animal proteins. The classic presentation of vitamin B12 deficiency is a combination of megaloblastic anemia and sub-acute combined degeneration of the cord and peripheral neuropathy. Up to $95 \%$ of patients with pernicious anemia have been reported to have neurological involvement, about a third of these experienced only mild symptoms such as paraesthesia of the hands and feet. This can eventually progress to include sensory loss, gait ataxia, distal limb weakness and ataxic paraplegia. In the early stage of the disease, the spinal cord appears to be affected first. ${ }^{3}$ The extent of involvement usually includes the posterior and lateral columns of the lower cervical and upper thoracic spinal cord in a contiguous way. The predominant involvement of the posterior columns of the spinal cord results in impairment of position and vibration sense and dysaethesia.

Vitamin B12 (cyanocobalamin) is found essentially in all meat and dairy products. It is an essential vitamin for DNA and fatty acid synthesis, including myelin synthesis. ${ }^{4}$ Vitamin B12 deficiency can be caused by gastro-intestinal malabsorption, poor nourishment of food or genetic deficiency of methylmalonyl-CoA mutase. 
The diagnosis of Vitamin B12 deficiency is confirmed by a low serum B12 level or elevation of its metabolites (homocysteine and methylmalonic acid). Results of MRI of the spine can be normal or non-specific. Also MRI of the Cervical and Dorsal spine shows increased T2 weighted signal intensity in the posterior and lateral columns of the cervical and upper thoracic spinal cord..$^{5-6}$ Both gadolinium enhancement and spinal cord swelling have been described. ${ }^{7}$ There was involvement of dorsal column as shown in our case (figure-3).

Treatment of Vitamin B12 deficiency depends on the cause and includes Cyanocobalamin 1000 microgram I/M daily for 1 week, followed by weekly injection for 1 month, followed by monthly for life.

\section{Conclusion}

Early diagnosis of sub acute combined degeneration of the spinal cord is important because the condition is curable with prompt replacement therapy. The degree of resolution of clinical symptoms is inversely proportional to the duration and severity of vitamin B12 deficiency. ${ }^{8}$ In the event of delayed treatment, irreversible disabling neurological impairment may occur. In our country though most of the people are non vegetarian, still there is the possibility of Vitamin B12 deficiency. Its diagnosis should be entertained if different parts of neural axis i.e. brain, spinal cord, optic nerve, peripheral nerves are involved; although there are few reported cases in our country.

\section{References}

1) Kalita J.Misra UK. Vitamin B12 deficiency neurological syndromes: correlation of clinical, MRI and cognitive evoked potential. J Neurol. 2008;255:353-9.

2) Jewesburg Eric CO. Subacute degeneration of the cord and achorhydria peripheral neuropathies without anaemia. Lancet. 1954;307-12.

3) So YT, Simon RP. Deficiency disease of the nervous system. Vitamin B12 deficiency. Neurology in clinical practice. $4^{\text {th }}$ ed. Bradley WG, Daroff RB, Fenichel GM, editors. Philadelphia (PA): Butterworth-Heinemann; 2004. p. 1594-7.

4) Wadia RS,Bandishri S,Kharche Vit.B12 and folate deficiency; incidence and clinical features. Neurol.India. 2000;48(4):302-4 .

5) Bassi SS, Bulundewee KK, Greef GP, Labuscagne $\mathrm{JH}$, Gledhill RF. MRI of the spinal cord in myelopathy complicating vitamin B12 deficiency: two additional cases and a review of the literature. Neuroradiology. 1999;41(4):271-4.

6) Grossman RI, Yousem DM. Neurodegerative diseases of the spine. Subacute combined degeneration (SCD). Neuro-Radiology. Philadelphia (PA). Mosby; 2003. p. 804-5.

7) Locatelli ER., Laureno R., Ballard. MRI in vitamin B12 deficiency myelopathy. Can J Neurol Sci. 1999;26:60-63.

8) Samuels MA, Feske S. Office practice of neurology. New York: Churchill Livingstone. 1996; p. 1009-13. 